\title{
Green Computing and Strategies for Energy Efficient Cloud Management
}

\author{
Rahul Nijhawan \\ M-Tech Research Scholar \\ Department of Computer \\ Science \& Engineering \\ HCTM Kaithal
}

\author{
Mukesh Rana, PhD \\ Guide \\ Assist. Professor, \\ Department of Computer \\ Science \& Engineering \\ HCTM Kaithal
}

\begin{abstract}
The term Green Computing relates to processing this is certainly eco-friendly it's also termed as green IT. Green computing is renewable to environment as the significant objectives are to reduce the usage dangerous products, maximizing energy efficiency throughout the product's lifetime, and advertise the recyclability or biodegradability of factory waste. Green processing also focuses on reducing the resource usage and disposing this is certainly accountable of waste. The investigation on cloud processing is still at an early on stage although cloud computing has quickly appeared as a widely accepted computing paradigm. Cloud suffers that are computing different challenging problems pertaining to security, computer software frameworks, quality of solution, standardization, and power usage. Efficient energy management is just one of the most researches that is challenging. The core services in cloud computing system are the SAAS (computer software as an ongoing solution), PAAS (Platform as a site), and IAAS (Infrastructure as a Service). In this report, we study state-of-the art strategies and analysis pertaining to power conserving in the IAAS of a cloud computing system, which consumes an enormous element of total power in a cloud system that is computing. Some possible solutions for building green cloud processing are proposed at the conclusion. Our aim is to supply an improved knowledge of the look difficulties of energy management into the IAAS of a cloud system that is processing.
\end{abstract}

\section{Keywords}

Green Cloud Computing, Energy Conservation, Dynamic Voltage Frequency Scaling.

\section{INTRODUCTION}

Large-scale companies and logical requests, that are normally composed of big-data, multitasking, time-variant, and fluctuating workloads [1], have come to be the mainstream of present technologies. For instance, Hardtop has been joined by Amazon alongside cloud computing shouted Amazon EMR that provides cloud ability for people that permits users swiftly and facilely grasp colossal numbers of data. With the quick development of data scale, employing the cloud computing knowledge to deal alongside varied requests has come to be increasingly important. Cloud computing, alongside virtualization as the key enabling knowledge, provides a flexible scaling-up and scaling down provisioning mechanism. Realization of these methods is established on the colossal scale of cloud data centers. And the elevated worth of power consumption has come to be a critical subject for these data centers.

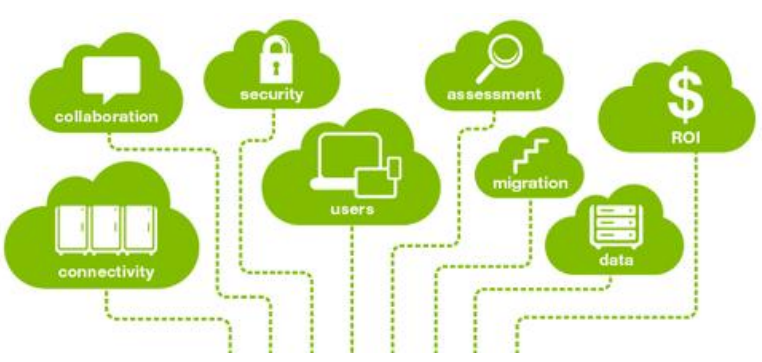

Fig 1: Architecture and Aspects of Green Cloud Computing

During the last insufficient years, the elevated worth of power consumption has come to be a critical issue. One of the research estimates that a data center alongside 50,000 computing nodes could use extra than one hundred million $\mathrm{kHz} /$ year equivalent to the electricity consumption for a 100,000 populated city in one year. The power consumption in data centers will yet rise swiftly in the subsequent ten years. On the supplementary hand, the CPU utilization for severs is moderately not high. The work in displays that the average CPU utilization of extra than 5,000 servers across a six-month era is amid 10 and 50 percent of their maximum utilization levels. These researches illuminate the PUE (Power Usage Effectiveness [2]: all power use of data center and IT burden consumption ratio) of most datacenters are unsatisfactory. Obviously, the criterions to assess mechanisms for parallel requests merely focus on minimizing the design length, but scarcely encounter the producing advocacy for green computing system. This paper considers that effectual task arranging in cloud nature ought to not merely endeavor to attain a negligible completion period but additionally rise the arrangement resource utilization as well as cut the power consumptions.

Based on the green computing believed, growing energyefficient mechanisms for parallel requests becomes increasingly attractive. The setbacks of parallel request arranging are NP-hard [3] in the finished case. Most of the static arranging setbacks can be resolved by a request embodied as Directed Acyclic Graph (DAG) [4] arranging, comparable to the work of Braun in that nodes stand for request tasks and borders embody inter task data dependencies. Moreover, assorted supplementary mechanisms for cutting the power consumption have been investigated in the preceding works, such as Dynamic Voltage/Frequency Scaling (DVFS) [5]. DPM turns the inactive constituents off managing the resources to the hibernate mode to cut the manipulation consumption. As it merely works after the inactive period is long plenty, DVFS has been proven to be a extremely enthusing method alongside its clarified skill for 
power savings. It is established on the fact that power consumption in CMOS routes has a manage connection alongside the square of the supplied voltage and frequency a colossal reduction in manipulation consumption can be attained by switching amid processor's voltages/frequencies across task killing as promising a little performance. Though, most of these ways are challenged alongside the fact that joining optimum to every single sub-problem could flout the globe optimality for the critical arrangement performance. In supplement to DVFS method, if requests are not time-critical, we can ponder minimizing the number of utilized processors by seizing supremacy of the inactive processor period amid the running tasks in parallel to rise the resource utilization. In this method, users could demand to tolerate a slight stay of killing for cutting arrangement power consumption. Thus, discovering the inefficient processors and curving them off joining the DVFS method could be a enthusing way to cutting power dissipation as well as promising the performance [6].

Cloud computing is an accumulation community based solutions offering you with on-demand, ubiquitous, convenient system access to a shared pool of configurable computing resources that may be rapidly provisioned and released in an easy and universal means with reduced management effort or Cloud Service Provider interaction. It offers solution that is new with additional resource that is efficient, on-demand scalability, and price decrease for organizations. Cloud computing has quickly evolved through various phases that consist of grid and utility computing and application service provisioning. These solutions are scaled along with regards to the client's adjustable operation's needs, ensuring maximum price efficiency. Use of cloud-based services enable companies to keep rate using the evolving that is quick company that is powerful, also as benefiting better reliability, less maintenance and higher accessibility. Oldfashioned access practice to usually cloud services is maybe not energy that is efficient. Companies cloud that is using lacks policies and decision making guidelines to enforce the administration that is overall reduction of energy usage, thus relying only on ad-hoc end-user's responsiveness to utilize the energy-saving schemes installed inside their computing infrastructure. Furthermore, a cutback in the vigor spending plan of a data center, without having to sacrifice service degree agreements, is an objective that is important well as economic incentive for data center operators. In this work that is ongoing we focus on optimizing client-side requests to cloud services, in order to reach reduced power consumption locally, therefore protecting the surroundings with lower carbon impact whenever access cloud services. We use a power that is simplified model centered on cloud services accessed for employment that is specific. The solution that is perfect proposed is an Integrated Green Cloud Architecture (IGCA) [7]. It includes a good client oriented 'Green Cloud Middleware' that may smooth the transition from main-stream (local) computing to cloud computing energy that is using that is cheapest, but susceptible to satisfying the service level agreement, keeping the product quality of service and remaining within the budget. Network-based cloud computing is rapidly expanding as an alternative to computing that is main-stream is office-based. As cloud becomes that are computing extensive, the energy consumption for the grouped community and computing resources that underpin the cloud will grow. This is happening at an occasion if you learn attention that is increasing paid to the requirement to manage energy usage throughout the knowledge sector. While data center energy use has gotten attention that is much, there is less attention paid towards the power use for the transmission and systems that are switching are foundational to to connecting users to the cloud. An analysis is presented by us of energy consumption in cloud computing in this paper. The analysis considers both public and clouds which can be private and includes energy consumption in switching and transmission since well as information processing and information space for storage. We reveal that power consumption in transport and switching can be a percentage that is significant of energy usage in cloud computing. Cloud computing can enable more use that is energy-efficient of energy, especially whenever the computing tasks are of low infrequent or intensity. Nevertheless, under some circumstances cloud computing can digest more power than conventional computing where each user does all computing on their laptop that is own or (PC). As we have actually discussed above that all architectures that are current some constructive along with destructive points. In this work we gave the architecture for green cloud the main advantageous asset of this architecture is emission that is co2, this directory steps the best solution that is suitable gives less carbon emission so instantly it indicates that energy will also decrease because $\mathrm{Co} 2$ emission and energy consumption both are straight proportionate to at least one another. Likewise the disadvantage is that only emission that is energy that is $\mathrm{CO} 2$ isn't the factor become under consideration like Quality Provisioning, Security, etc. [8] [9] [10].

In this paper, established on encounter the presentation established ability level accord, we counsel a new power Cognizant arranging algorithm shouted DVF Enabled Efficient-energy Workflow Task Arranging to optimize the power savings across DVFS method for parallel requests in the heterogeneous distributed computing systems. In this paper, the result and presentation of DVFS are approximated across comprehensive examinations, below the maximum presentation conditions, disparate number of processors, assorted expansion ratios, disparate benefits of CCR, and disparate degree of parallelism. And the assessing indexes are four presentation metrics: power consumption ratio (ECR), arrangement resource utilization ratio, average killing period, and power saving ratio. The main contributions of this paper are summarized below.

1. This paper proposes an energy-aware task scheduling algorithm. Within a given deadline, this algorithm can distribute the parallel applications in workflows to appropriate processors, and deals with them at the appropriate time slots to reduce energy consumption as well as meeting the required performance.

2. Numerical experiments are given to verify that DVFS can increase the CPU utilization of processors and reduce significant amount of energy consumption in a wide range of workflow structures compared with other researches.

3 . We analyze the factors which are affecting the performance of our algorithm.

\section{OVERVIEW OF ENERGY AWARE TECHNIQUES FOR CLOUD}

Current Web requests demand exceedingly flexible hosting and resource provisioning solutions. The rising popularity of communal web locations, and the desire of present Internet users to store and allocate rising numbers of data (e.g. pictures, movies, life-stories, and adjacent farms) have needed scalable infrastructure. Benefiting from economies of scale and present events in Web technologies, data centers have appeared as a key ideal to ability resources to Web requests 
and deal alongside their potential and presentation requirements. Though, data centers are frequently provisioned to grasp sporadic top loads that can consequence in low resource utilization and wastage of energy.

The ever-increasing demand for cloud-based services does rise the alarming concern of data center power consumption. Current reports indicate that power prices are becoming dominant in the Finished Price of Ownership (TCO). In 2006, data centers embodied 1.5 percent of the finished US electricity consumption. By 2011, the present data center power consumption might double lead to extra carbon emissions. Electricity becomes the new manipulating factor for employing data center equipment's.

A scope of technologies can be utilized to make cloud computing infrastructures extra power effectual, encompassing larger cooling technologies, temperature-aware arranging, Dynamic Voltage and Frequency Scaling (DVFS) and resource virtualization. The use of VMs brings countless benefits encompassing nature and presentation isolations; enhanced resource utilization by enabling workload consolidation; and resource provisioning on demand. Nevertheless, such technologies ought to be analyzed and utilized prudently for truly enhancing the energy-efficiency of computing infrastructures. Consolidation algorithms have to deal alongside the connection amid presentation, resource utilization and power, and can seize supremacy from resource heterogeneity and request affinities. Additionally, methods such as VM live-migration can considerably enhance the capacities of Cloud settings by enabling obligation association, burden balancing and lowering arrangement maintenance costs. VM migration provides a extra flexible and flexible resource association and proposals a new period of virtualization by removing the believed of locality in virtualized environments.

The overhead acted by VM technologies has cut above the years, that has increased their appeal for running elevated presentation computing requests and coiled virtualization into a mainstream knowledge for grasping and bestowing resources for a expansive user area alongside heterogeneous software-stack requirements. VM-based resource association arrangements such as Eucalyptus and Open Nebula permit users to instantiate and customize clusters of adjacent mechanisms atop the underlying hardware infrastructure. After requested in a data center nature, virtualization can permit for amazing workload consolidation. For instance, as Web requests normally present variable user populace and period variant workloads, virtualization can be retained to cut the power consumed by the data center nature across server consolidation whereby VMs running disparate workloads can allocate the alike physical host. By joining the workload of user requests into less mechanism, new servers can potentially be switched off or locale in low power consumption modes. Yet appealing virtualization is, it's merely use does not promise reductions in power consumption. Enhancing the power efficiency of Cloud settings alongside the assistance of virtualization usually calls for arranging mechanisms that adaptively ability requests alongside resources that match their workload demands and utilizes supplementary manipulation association technologies such as CPU throttling and Dynamic reconfiguration; permitting new resources to be freed or switched off.

Existing work has counseled architectures that benefit from virtualization for making data centers and Clouds extra power efficient. The setback of energy-efficient resource provisioning is usually tear into two sub setbacks at micro-or host level, manipulation association methods are requested to minimize the number of resources utilized by requests and hence cut the power consumed by an individual host; and at a macro-level, usually a Resource Association Arrangement (RMS) strives to impose arranging and workload consolidation strategies that endeavor to cut the number of nodes needed to grasp the workloads of user requests or locale requests in spans of a data center that should enhance the effectiveness of the cooling system. A little of the methods and data usually investigated and requested at a macro- or RMS-level to accomplish workload consolidation.

Server consolidation has been investigated in preceding work. A key constituent of these arrangements is the skill to monitor and guesstimate the workload of requests or the entrance of user requests. Countless methods have been requested to guesstimate the burden of a arrangement, such as exponential advancing averages Kaman filters autoregressive models, and combinations of methods. Provisioning VMs in an Iasi nature poses supplementary trials as data concerning the user requests is not always effortlessly available. Serving 4.3 describes an algorithm for forecasting the characteristics of advance reservation demands that resemble demands for allocating adjacent machines.

Fitted alongside workload-estimation methods, these arrangements furnish schemes to minimize the power consumed by the underlying groundwork as minimizing prices and violations of Service Level Accords (SLAs). Pursue et al. gave MUSE, an economy-based arrangement that allocates resources of hosting centers to services aiming to minimize power consumption. Autonomic resource provisioning employing Kaman filters. Music et al. counseled a gaze in front manipulation scheme for steadily optimizing the manipulation efficiency of a virtualized environment. With the aim of maximizing the profit yielded by the arrangement as minimizing the manipulation consumption and SLA violations, the provisioning setback is modeled as a sequential optimization below uncertainty and is resolved employing the gaze in front manipulation scheme. Arrangement of requests and arranging can additionally seize into report the thermal states or the warmth dissipation in a data center. The aim is arranging workloads in a data center, and the warmth they produce, in a manner that minimizes the power needed by the cooling groundwork, hence aiming to minimize prices and rise the finished reliability of the platform.

Although consolidation fitted alongside burden forecasting schemes can cut the finished number of resources utilized to assist user requests, the deeds gave by RMSs to change the nature to match the request demands can need the relocation and reconfiguration of VMs. That can encounter the reply period of requests, subsequently degrading the QOS observed by conclude users. Hence, it is vital to ponder the prices and benefits of the adaptation actions. For example, Guiyang et al. have discovered a cost-sensitive adaptation engine that weights the possible benefits of reconfiguration and their costs. A price ideal for every single request is crafted offline and to choose after and how to reconfigure the VMs, the adaptation engine estimates the price of adaptation deeds in words of adjustments in the utility, that is a purpose of the request reply time. The benefit of an deed is given by the enhancement in request reply period and the era above that the arrangement stays in the new configuration.

Moreover, consolidation raises the subject of dealing alongside vital redundancy and arrangement geo-diversity at the alike time. Cloud providers, as Salesforce.com for 
example, that proposal to host whole websites of confidential firms, do not desire to lose whole firm websites because of manipulation outages or web admission failures. Hence, outage and blackout situations ought to be anticipated and seized into report in the resource association policies.

While the macro-level resource association performs deeds that usually seize into report the manipulation consumption of a cluster of resources or the finished data center, at the hostlevel the manipulation association is gave by configuring parameters of the hypervisor's scheduler, such as throttling of Adjacent CPUs (VCPU) and employing supplementary OS specific policies. In the counseled architectures, hosts usually run a innate resource manager that is accountable for monitoring the manipulation consumption of the host and optimizing it according to innate policies. The manipulation association skills obtainable in virtualized hosts have been categorized as: "soft" deeds such as CPU idling and throttling; "hard" deeds like DVFS; and joining in the hypervisor. CPU idling or soft states encompass in changing resource allotments of VMs and qualities of the hypervisor's scheduler (e.g. number of credits in XEN's trust scheduler) to cut the $\mathrm{CPU}$ period allocated to a VM so that it consumes less power. Hard deeds contain methods such as scaling the voltage and frequency of CPUs. Consolidation can additionally be gave at the host-level whereas the VCPUs allocated to VMs can be configured to allocate CPU cores, allocating new cores in inactive state, hence saving the power that should or else be utilized by the supplementary core to run a VM.

Nathuji and Schwann gave Adjacent Power, a manipulation association arrangement for virtualized settings that discover both hardware manipulation scaling and software-based methods to manipulation the manipulation consumption of underlying platforms. Adjacent Domination exports a set of manipulation states to VM visitors that permit visitors to use and deed on these states therefore giving their own manipulation association policies. The soft states are interrupted by XEN hypervisor and are mapped to adjustments in the underlying hardware such as CPU frequency scaling according to the adjacent manipulation association rules. The manipulation association strategies requested in the visitor VMs is utilized as "hints" by the hypervisor rather than executable commands. They additionally assess the manipulation drawn by cores at disparate frequency/voltage levels and counsel that such method be utilized alongside soft schemes.

\section{DVFS (DYNAMIC VOLTAGE FREQUENCY SCALING)}

As DVFS method can be requested by monitoring the CPU utilization. After the workload is heavy, real-time migration can be endowed for accomplished extra competent custom of resources below the user unaware situation. Dynamic voltage frequency scaling is a hardware knowledge that can dynamically adjust the voltage and frequency of the processor in time [11]. By requesting DVFS knowledge, lacking possessing to restart the manipulation supply, arrangement voltage and frequency can be adjusted in accordance alongside the specification of the early CPU design into a disparate working voltage. As CPU works in lower voltage, the power consumption can efficiently be saved.

The manipulation consumption of the CPU is measured by increasing the voltage square alongside the arrangement frequency. Whereas $\mathrm{V}$ is the voltage, $\mathrm{F}$ is the frequency, and $\mathrm{C}$ is the capacitive burden of the system. The DVFS is the manipulation saving knowledge by cutting the voltage supply.
The reduction of CPU frequency way that the voltage can additionally be dropped, nevertheless it wills consequence in the degradation of the arrangement presentation and lead to prolong the killing time. In supplement, the overhead of the voltage adjusting ought to additionally be considered. The intention of the DVFS is to permit the filth of the killing speed of a task by cutting the CPU frequency and voltage to cut the manipulation blazing up. This knowledge is frequently utilized in real-time systems.

$$
\mathrm{P}=\mathrm{V}^{2} \times \mathrm{A} \times \mathrm{F} \times \mathrm{C}
$$

As remarked above, by requesting the DVFS knowledge, CPU voltage can be lowered, but the killing speed of the task will be reduced. From Equation, we can discern that if merely cutting the frequency, power cannot be saved effectively. In the arrangement, $\mathrm{C}$ is the capacitive burden of the arrangement, merely in lowering the frequency and additionally cutting the voltage, the manipulation consumption can be saved effectively. A is the main controller factor for frequency which is updated by number of task user submits.

Figure below show the results of proposed DVFS based policy compared with Non DVFS policy and its effectiveness in energy saving.

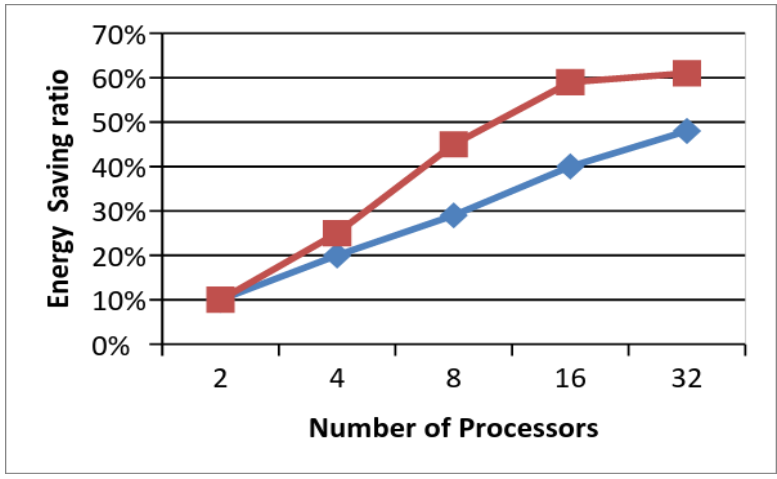

\section{Fig 2: Energy Saving Ratio Proposed DVFS based policy compared with Non DVFS}

For each digital device to be allocated in the datacenter its checked on all hosts which are the ones that can have the incoming machine that is virtual. The condition that is primary a number to receive a virtual machine is having sufficient MIPS available for the VM to be allocated. The effectiveness of energy that it consumes is confirmed for each candidate host. The algorithm ranks the host that is more power efficient to allocate the machine that is virtual machine. A tie can especially occur in homogeneous environments. A tiebreaker comparison is made as follows: considering that the functional system has DVFS enabled, along with CPU Control, there will likely be exist some hosts consuming less energy than others. So, hosts are confronted against each other and the smaller energy consumer is selected to allocate the machine that is a virtual machine. Yet there may be a tie, for example if there are two or more machines of equal power consumption that still haven't received machines which can be virtual. Therefore another tiebreaker is performed: the host because of the CPU utilization that is highest is chosen. This choice is dependent on the fact that when a CPU is processing more load, it will take longer to achieve its load that is digital migration. The host with more processing power is chosen, since this is potentially in a position to receive a larger 
number of workloads if there was still a tie. Figure below shows the Average Resource Utilization per processor of Proposed DVFS based policy (blue) at minimal Task load, (red) at normal task loads, (green) at HPC (high performance computing).

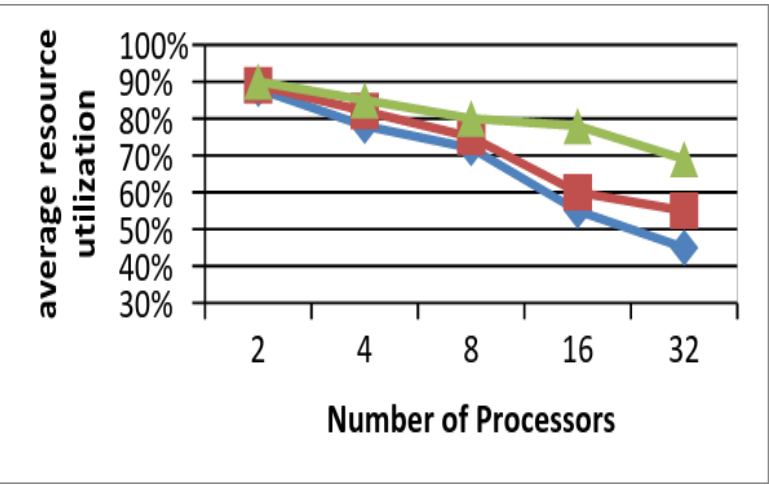

Fig 3: Average Resource Utilization per processor of Proposed DVFS based policy

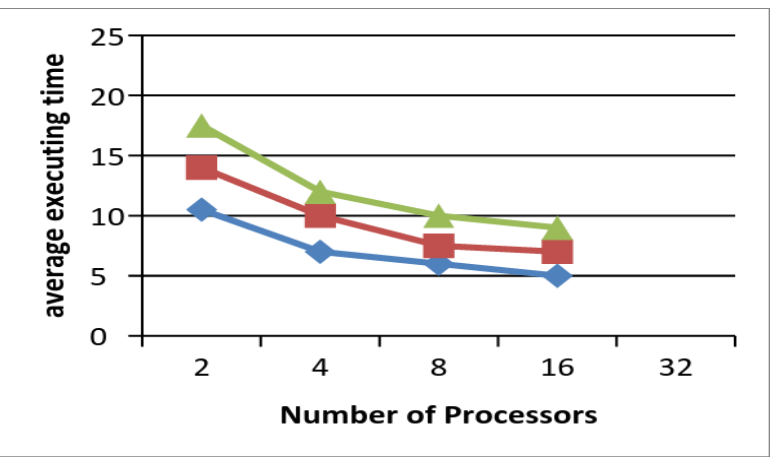

Fig 4: Average Executing Time Proposed DVFS based policy for three scenarios

Figure above shows the Average Execution time per processor of Proposed DVFS based policy (blue) at minimal Task load, (red) at normal task loads, (green) at HPC (high performance computing).

Once all the machines which are digital been produced, the loads are submitted for processing. The loads that could not be processed will be delayed if you can find insufficient virtual machines available for processing such loads. The hosts and turns off virtual machines which may have completed the processing of these loads with all loads submitted the algorithm checks. The algorithm checks which hosts are being utilized below a defined threshold and attempts to migrate the virtual machines from these hosts to other ones, and then shut down the underutilized hosts at this point. The selection of target host in the migrations is completed with the algorithm that is same. The worth that is optimal of could be difficult to calculate. You will find academic researches on which would end up being the means that is better to calculate this threshold. The application of a colony that is a honeybee calculates the good thresholds for virtual load migration. As future work we consider incorporating strategies like this to implement thresholds which are adaptive our algorithm.

\section{CONCLUSION \& FUTURE WORK}

Accurate vision of the upcoming workload permits the datacenter operator to locale unneeded physical servers in a low-power state to save energy. If extra arrangement capacity is needed, servers in a low-power state are transitioned back to an alert state. In this paper, we spread our prior research by giving a new way to ascertaining the frequency of computing the forecast of the anticipated capacity. We present a Dynamic forecast quantization method to ascertain the optimal number of forecast calculation intervals. These new algorithms permit us to forecast upcoming burden inside needed Service Level Accords as minimizing the number of periods the forecast calculations have to be performed. The main goal of this research work was to join the Dynamic Voltage Frequency Scaling method in an effectual method to maximize the resource utilization and minimize power consumption of the data center. Minimizing Power can consequence in the less number of carbon impressions and therefore will aid extra in accomplished Green Computing. Our Aftermath display that the DVFS established Cloud Calculating we have industrialized can produce extra than $40-53 \%$ effectual aftermath above virtualized cloud arrangement.

\section{REFERENCES}

[1] Armbrust, Michael, Armando Fox, Rean Griffith, Anthony D. Joseph, Randy Katz, Andy Konwinski, Gunho Lee et al. "A view of cloud computing." Communications of the ACM 53, no. 4 (2010): 50-58.

[2] Zhang, Qi, Lu Cheng, and Raouf Boutaba. "Cloud Computing: state-of-the-art and research challenges." Journal of internet services and applications 1, no. 1 (2010): 7-18

[3] Pandey, Suraj, Linlin Wu, Siddeswara Mayura Guru, and Rajkumar Buyya. "A particle swarm optimization-based heuristic for scheduling workflow applications in cloud computing environments." In Advanced information networking and applications (AINA), 2010 24th IEEE international conference on, pp. 400-407. IEEE, 2010.

[4] Bessai, Kahina, Samir Youcef, Ammar Oulamara, Claude Godart, and Selmin Nurcan. "Bi-criteria workflow tasks allocation and scheduling in Cloud Computing Environments. "In Cloud Computing (CLOUD), 2012 IEEE 5th International Conference on, pp. 638-645. IEEE, 2012.

[5] Shen, Zhiming, Sethuraman Subbiah, Xiaohui Gu, and John Wilkes. "Cloudscale: elastic resource scaling for multi-tenant cloud systems." In Proceedings of the 2nd ACM Symposium on Cloud Computing, p. 5. ACM, 2011.

[6] Baliga, Jayant, Robert WA Ayre, Kerry Hinton, and Rodney S. Tucker. "Green cloud computing: Balancing energy in processing, storage, and transport." Proceedings of the IEEE 99, no. 1 (2011): 149-167.

[7] Hulkury, Mohammad Naiim, and Mohammad Razvi Doomun. "Integrated green cloud computing architecture." In Advanced Computer Science Applications and Technologies (ACSAT), 2012 International Conference on, pp. 269-274. IEEE, 2012.

[8] Buyya, Rajkumar, Anton Beloglazov, and Jemal Abawajy. "Energy-efficient management of data center resources for cloud computing: a vision, architectural elements, and open challenges." arXiv preprint arXiv:1006.0308 (2010). 
International Journal of Computer Applications (0975 - 8887)

Volume 150-No.2, September 2016

[9] Garg, Saurabh Kumar, Chee Shin Yeo, and Rajkumar Buyya. "Green cloud framework for improving carbon efficiency of clouds." In Euro-Par 2011 Parallel Processing, pp. 491-502. Springer Berlin Heidelberg, 2011.

[10] Garg, Saurabh Kumar, and Rajkumar Buyya. "Green cloud computing and environmental sustainability."
Harnessing Green IT: Principles and Practices (2012): 315-340.

[11] Kliazovich, Dzmitry, Pascal Bouvry, and Samee Ullah Khan. "GreenCloud: a packet-level simulator of energyaware. 